\title{
8 How Precise Are Price Distributions Predicted by Implied Binomial Trees?
}

Wolfgang Härdle and Jun Zheng

In recent years, especially after the 1987 market crash, it became clear that the prices of the underlying asset do not exactly follow the Geometric Brownian Motion (GBM) model of Black and Scholes. The GBM model with constant volatility leads to a log-normal price distribution at any expiration date: All options on the underlying must have the same Black-Scholes (BS) implied volatility, and the Cox-Ross-Rubinstein (CRR) binomial tree makes use of this fact via the construction of constant transition probability from one node to the corresponding node at the next level in the tree. In contrast, the implied binomial tree (IBT) method simply constructs a numerical procedure consistent with the volatility smile. The empirical fact that the market implied volatilities decrease with the strike level, and increase with the time to maturity of options is better reflected by this construction. The algorithm of the IBT is a data adaptive modification of the CRR method.

An implied tree should satisfy the following principles:

- It must correctly reproduce the volatility smile.

- negative node transition probabilities are not allowed.

- The branching process must be risk neutral (forward price of the asset equals to the conditional expected value of it) at each step .

The last two conditions also eliminate arbitrage opportunities.

The basic purpose of the IBT is its use in hedging and calculations of implied probability distributions (or state price density (SPD)) and volatility surfaces. 
Besides these practical issues, the IBT may evaluate the future stock price distributions according to the BS implied volatility surfaces which are calculated from currently observed daily market option prices.

We describe the construction of the IBT and analyze the precision of the predicted implied price distributions. In Section 8.1, a detailed outline of the IBT algorithm for a liquid European-style option is given. We follow first the Derman and Kani (1994) algorithm, discuss its possible shortcomings, and then present the Barle and Cakici (1998) construction. This method is characterized by a normalization of the central nodes according to the forward price. Next, we study the properties of the IBT via Monte-Carlo simulations and comparison with simulated conditional density from a diffusion process with a non-constant volatility. In Section 8.3, we apply the IBT to a DAX index data set containing the underlying asset price, strike price, interest rate, time to maturity, and call or put option price from the MD*BASE database (included in XploRe), and compare SPD estimated by historical index price data with those predicted by the IBT. Conclusions and discussions on practical issues are presented in the last section.

\subsection{Implied Binomial Trees}

A well known model for financial option pricing is a GBM with constant volatility, it has a log-normal price distribution with density,

$$
p\left(S_{t}, S_{T}, r, \tau, \sigma\right)=\frac{1}{S_{T} \sqrt{2 \pi \sigma^{2} \tau}} \exp \left[-\frac{\left\{\ln \left(\frac{S_{T}}{S_{t}}\right)-\left(r-\frac{\sigma^{2}}{2}\right) \tau\right\}^{2}}{2 \sigma^{2} \tau}\right],
$$

at any option expiration $T$, where $S_{t}$ is the stock price at time $t, r$ is the riskless interest rate, $\tau=T-t$ is time to maturity, and $\sigma$ the volatility. The model also has the characteristic that all options on the underlying must have the same BS implied volatility.

However, the market implied volatilities of stock index options often show "the volatility smile", which decreases with the strike level, and increases with the time to maturity $\tau$. There are various proposed extensions of this GBM model to account for "the volatility smile". One approach is to incorporate a stochastic volatility factor, Hull and White (1987); another allows for discontinuous jumps in the stock price, Merton (1976). However, these extensions cause several practical difficulties. For example, they violate the risk-neutral condition. 
The IBT technique proposed by Rubinstein (1994), Derman and Kani (1994), Dupire (1994), and Barle and Cakici (1998) account for this phenomenon. These papers assume the stock prices in the future are generated by a modified random walk where the underlying asset has a variable volatility that depends on both stock price and time. Since the implied binomial trees allow for nonconstant volatility $\sigma=\sigma\left(S_{t}, t\right)$, they are in fact modifications of the original Cox, Ross and Rubinstein (1979) binomial trees. The IBT construction uses the observable market option prices in order to estimate the implied distribution. It is therefore nonparametric in nature. Alternative approaches may be based on the kernel method, Aït-Sahalia, and Lo (1998), nonparametric constrained least squares, Härdle and Yatchew (2001), and curve-fitting methods, Jackwerth and Rubinstein (1996).

The CRR binomial tree is the discrete implementation of the GBM process

$$
\frac{d S_{t}}{S_{t}}=\mu d t+\sigma d Z_{t},
$$

where $Z_{t}$ is a standard Wiener process, and $\mu$ and $\sigma$ are constants. Similarly, the IBT can be viewed as a discretization of the following model in which the generalized volatility parameter is allowed to be a function of time and the underlying price,

$$
\frac{d S_{t}}{S_{t}}=\mu_{t} d t+\sigma\left(S_{t}, t\right) d Z_{t},
$$

where $\sigma\left(S_{t}, t\right)$ is the instantaneous local volatility function. The aim of the IBT is to construct a discrete approximation of the model on the basis of the observed option prices yielding the variable volatility $\sigma\left(S_{t}, t\right)$. In addition, the IBT may reflect a non-constant drift $\mu_{t}$.

\subsubsection{The Derman and Kani (D \& K) algorithm}

In the implied binomial tree framework, stock prices, transition probabilities, and Arrow-Debreu prices (discounted risk-neutral probabilities, see Chapter 9) at each node are calculated iteratively level by level.

Suppose we want to build an IBT on the time interval $[0, T]$ with equally spaced levels, $\Delta t$ apart. At $t=0, S_{0}=S$, is the current price of the underlying, and there are $n$ nodes at the $n$th level of the tree. Let $s_{n, i}$ be the stock price of the $i$ th node at the $n$th level, $s_{1,1}=S$ and $F_{n, i}=e^{r \Delta t} s_{n, i}$ the forward price at level $n+1$ of $s_{n, i}$ at level $n$, and $p_{n, i}$ the transition probability of making 


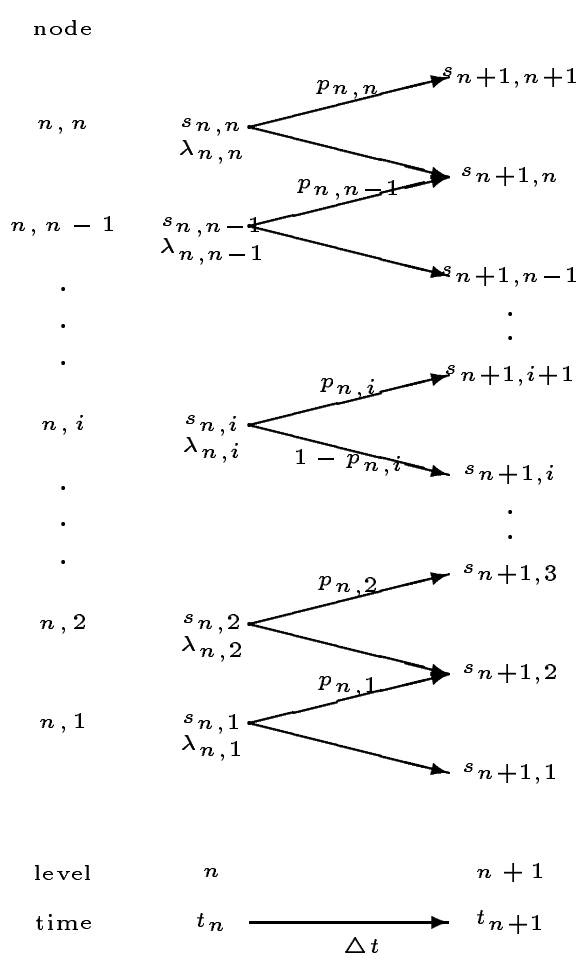

Figure 8.1. Construction of an implied binomial tree

a transition from node $(n, i)$ to node $(n+1, i+1)$. Figure 8.1 illustrates the construction of an IBT.

We assume the forward price $F_{n, i}$ satisfies the risk-neutral condition:

$$
F_{n, i}=p_{n, i} s_{n+1, i+1}+\left(1-p_{n, i}\right) s_{n+1, i} .
$$

Thus the transition probability can be obtained from the following equation:

$$
p_{n, i}=\frac{F_{n, i}-s_{n+1, i}}{s_{n+1, i+1}-s_{n+1, i}} .
$$

The Arrow-Debreu price $\lambda_{n, i}$, is the price of an option that pays 1 unit payoff in one and only one state $i$ at $n$th level, and otherwise pays 0 . In general, 
Arrow-Debreu prices can be obtained by the iterative formula, where $\lambda_{1,1}=1$ as a definition.

$$
\left\{\begin{array}{l}
\lambda_{n+1,1}=e^{-r \Delta t}\left\{\left(1-p_{n, 1}\right) \lambda_{n, 1}\right\} \\
\lambda_{n+1, i+1}=e^{-r \Delta t}\left\{\lambda_{n, i} p_{n, i}+\lambda_{n, i+1}\left(1-p_{n, i+1}\right)\right\}, \quad 2 \leq i \leq n, \\
\lambda_{n+1, n+1}=e^{-r \Delta t}\left\{\lambda_{n, n} p_{n, n}\right\} .
\end{array}\right.
$$

We give an example to illustrate the calculation of Arrow-Debreu prices in a CRR Binomial tree. Suppose that the current value of the underlying $S=100$, time to maturity $T=2$ years, $\triangle t=1$ year, constant volatility $\sigma=10 \%$, and riskless interest rate $r=0.03$, and $\tau=T$. The Arrow-Debreu price tree can be calculated from the stock price tree:

stock price

$$
122.15
$$

110.52

100.00

100.00

90.48

81.88

Arrow-Debreu price

$$
\begin{array}{ccc} 
& & 0.37 \\
1.00 & 0.61 & \\
& 0.36 & 0.44 \\
& & 0.13
\end{array}
$$

For example, using the CRR method, $s_{2,1}=s_{1,1} e^{-\sigma \Delta t}=100 \times e^{-0.1}=90.48$, and $s_{2,2}=s_{1,1} e^{\sigma \Delta t}=110.52$, the transition probability $p_{1,1}=0.61$ is obtained by the formula (8.5), then according to the formula (8.6), $\lambda_{2,1}=e^{-r \Delta t}(1-$ $\left.p_{1,1}\right)=0.36$. At the third level, calculate the stock prices according to the corresponding nodes at the second level, For example, $s_{3,1}=s_{2,1} \cdot e^{-\sigma \Delta t}=$ $122.15, s_{3,2}=s_{1,1}=100$. 
Option prices in the Black-Scholes framework are given by:

$$
\begin{aligned}
& C(K, \tau)=e^{-r \tau} \int_{0}^{+\infty} \max \left(S_{T}-K, 0\right) p\left(S_{t}, S_{T}, r, \tau\right) d S_{T} \\
& P(K, \tau)=e^{-r \tau} \int_{0}^{+\infty} \max \left(K-S_{T}, 0\right) p\left(S_{t}, S_{T}, r, \tau\right) d S_{T}
\end{aligned}
$$

where $C(K, \tau)$ and $P(K, \tau)$ are call option price and put option price respectively, and $K$ is the strike price. In the IBT, option prices are calculated analogously for $\tau=n \triangle t$,

$$
\begin{aligned}
C(K, n \triangle t) & =\sum_{i=1}^{n+1} \lambda_{n+1, i} \max \left(s_{n+1, i}-K, 0\right), \\
P(K, n \triangle t) & =\sum_{i=1}^{n+1} \lambda_{n+1, i} \max \left(K-s_{n+1, i}, 0\right) .
\end{aligned}
$$

Using the risk-neutral condition (8.4) and the discrete option price calculation from (8.9) or (8.10), one obtains the iteration formulae for constructing the IBT.

There are $(2 n+1)$ parameters which define the transition from the $n$th to the $(n+1)$ th level of the tree, i.e., $(n+1)$ stock prices of the nodes at the $(n+1)$ th level, and $n$ transition probabilities. Suppose $(2 n-1)$ parameters corresponding to the $n$th level are known, the $s_{n+1, i}$ and $p_{n, i}$ corresponding to the $(n+1)$ th level can be calculated depending on the following principles:

We always start from the center nodes in one level, if $n$ is even, define $s_{n+1, i}=$ $s_{1,1}=S$, for $i=n / 2+1$, and if $n$ is odd, start from the two central nodes $s_{n+1, i}$ and $s_{n+1, i+1}$ for $i=(n+1) / 2$, and suppose $s_{n+1, i}=s_{n, i}^{2} / s_{n+1, i+1}=$ $S^{2} / s_{n+1, i+1}$, which adjusts the logarithmic spacing between $s_{n, i}$ and $s_{n+1, i+1}$ to be the same as that between $s_{n, i}$ and $s_{n+1, i}$. This principle yields the calculation formula of $s_{n+1, i+1}$, see Derman and Kani (1994),

$$
s_{n+1, i+1}=\frac{S\left\{e^{r \Delta t} C(S, n \triangle t)+\lambda_{n, i} S-\rho_{u}\right\}}{\lambda_{n, i} F_{n, i}-e^{r \Delta t} C(S, n \triangle t)+\rho_{u}} \quad \text { for } \quad i=(n+1) / 2 .
$$

Here $\rho_{u}$ denotes the following summation term

$$
\rho_{u}=\sum_{j=i+1}^{n} \lambda_{n, j}\left(F_{n, j}-s_{n, i}\right),
$$


$C(K, \tau)$ is the interpolated value for a call struck today at strike price $K$ and time to maturity $\tau$. In the $\mathrm{D} \& \mathrm{~K}$ construction, the interpolated option price entering (8.11) is based on a CRR binomial tree with constant parameters $\sigma=\sigma_{i m p}(K, \tau)$, where the BS implied volatility $\sigma_{i m p}(K, \tau)$ can be calculated from the known market option prices. Calculating interpolated option prices by the CRR method has a drawback, it is computational intensive.

Once we have the initial nodes' stock prices, according to the relationships among the different parameters, we can continue to calculate those at higher nodes $(n+1, j), j=i+2, \ldots n+1$ and transition probabilities one by one using the formula:

$$
s_{n+1, i+1}=\frac{s_{n, i}\left\{e^{r \Delta t} C\left(s_{n, i}, n \triangle t\right)-\rho_{u}\right\}-\lambda_{n, i} s_{n, i}\left(F_{n, i}-s_{n+1, i}\right)}{\left\{e^{r \Delta t} C\left(s_{n, i}, n \triangle t\right)-\rho_{u}\right\}-\lambda_{n, i}\left(F_{n, i}-s_{n+1, i}\right)},
$$

where the definition of $\rho_{u}$ is the same as (8.12).

Similarly, we are able to continue to calculate the parameters at lower nodes $(n+1, j), j=i-1, \ldots, 1$ according to the following recursion:

$$
s_{n+1, i}=\frac{s_{n, i+1}\left\{e^{r \Delta t} P\left(s_{n, i}, n \triangle t\right)-\rho_{l}\right\}-\lambda_{n, i} s_{n, i}\left(F_{n, i}-s_{n+1, i+1}\right)}{\left\{e^{r \Delta t} P\left(s_{n, i}, n \triangle t\right)-\rho_{l}\right\}+\lambda_{n, i}\left(F_{n, i}-s_{n+1, i+1}\right)},
$$

where $\rho_{l}$ denotes the sum $\sum_{j=1}^{i-1} \lambda_{n, j}\left(s_{n, i}-F_{n, j}\right)$, and $P(K, \tau)$ is similar to $C(K, \tau)$, again these option prices are obtained by the CRR binomial tree generated from market options prices.

\subsubsection{Compensation}

In order to avoid arbitrage, the transition probability $p_{n, i}$ at any node should lie between 0 and 1 , it makes therefore sense to limit the estimated stock prices

$$
F_{n, i}<s_{n+1, i+1}<F_{n, i+1} .
$$

If the stock price at any node does not satisfy the above inequality, we redefine it by assuming that the difference of the logarithm of the stock prices between this node and its adjacent node is equal to the corresponding two nodes at the previous level, i.e.,

$$
\log \left(s_{n+1, i+1} / s_{n+1, i}\right)=\log \left(s_{n, i} / s_{n, i-1}\right) .
$$


Sometimes, the obtained price still does not satisfy inequality (8.15), then we choose the average of $F_{n, i}$ and $F_{n, i+1}$ as a proxy for $s_{n+1, i+1}$.

In fact, the product of the Arrow-Debreu prices $\lambda_{n, i}$ at the $n$th level with the influence of interest rate $e^{r(n-1) \Delta t}$ can be considered as a discrete estimation of the implied distribution, the SPD, $p\left(S_{T}, S_{t}, r, \tau\right)$ at $\tau=(n-1) \triangle t$. In the case of the GBM model with constant volatility, this density is corresponding to $(8.1)$.

After the construction of an IBT, we know all stock prices, transition probabilities, and Arrow-Debreu prices at any node in the tree. We are thus able to calculate the implied local volatility $\sigma_{l o c}\left(s_{n, i}, m \Delta t\right.$ ) (which describes the structure of the second moment of the underlying process) at any level $m$ as a discrete estimation of the following conditional variance at $s=s_{n, i}, \tau=m \triangle t$. Under the risk-neutral assumption

$$
\begin{aligned}
\sigma_{l o c}^{2}(s, \tau) & =\operatorname{Var}\left(\log S_{t+\tau} \mid S_{t}=s\right) \\
& =\int\left(\log S_{t+\tau}-\mathrm{E} \log S_{t+\tau}\right)^{2} p\left(S_{t+\tau} \mid S_{t}=s\right) d S_{t+\tau} \\
& =\int\left(\log S_{t+\tau}-\mathrm{E} \log S_{t+\tau}\right)^{2} p\left(S_{t}, S_{t+\tau} r, \tau\right) d S_{t+\tau} .
\end{aligned}
$$

In the IBT construction, the discrete estimation can be calculated as:

$$
\sigma_{l o c}\left(s_{n, i}, \triangle t\right)=\sqrt{p_{n, i}\left(1-p_{n, i}\right)}\left|\log \frac{s_{n+1, i+1}}{s_{n+1, i}}\right| .
$$

Analogously, we can calculate the implied local volatility at different times. In general, if we have calculated the transition probabilities $p_{j}, j=1, \ldots, m$ from the node $(n, i)$ to the nodes $(n+m, i+j), j=i, \ldots, m$, then with

$$
\begin{array}{r}
\text { mean }=\mathrm{E}\left(\log \left(S_{(n+m-1) \Delta t}\right) \mid S_{(n-1) \triangle t}=s_{n, i}\right)=\sum_{j=1}^{m} p_{j} \log \left(s_{n+m, i+j}\right), \\
\sigma_{l o c}\left(s_{n, i}, m \triangle t\right)=\sqrt{\left.\sum_{j=1}^{m} p_{j}\left(\log \left(s_{n+m, i+j}\right)-m e a n\right)\right)^{2}} .
\end{array}
$$

Notice that the instantaneous volatility function used in (8.3) is different from the BS implied volatility function defined in (8.16), but in the GBM they are identical. 
If we choose $\Delta t$ small enough, we obtain the estimated SPD at fixed time to maturity, and the distribution of implied local volatility $\sigma_{l o c}(s, \tau)$. Notice that the BS implied volatility $\sigma_{i m p}(K, \tau)$ (which assumes Black-Scholes model is established (at least locally)) and implied local volatility $\sigma_{l o c}(s, \tau)$ is different, they have different parameters, and describe different characteristics of the second moment.

\subsubsection{Barle and Cakici (B \& C) algorithm}

Barle and Cakici (1998) proposed an improvement of the Derman and Kani construction. The major modification is the choice of the stock price of the central nodes in the tree: their algorithm takes the riskless interest rate into account. If $(n+1)$ is odd, then $s_{n+1, i}=s_{1,1} e^{r n \Delta t}=S e^{r n \Delta t}$ for $i=n / 2+1$, if $(n+1)$ is even, then start from the two central nodes $s_{n+1, i}$ and $s_{n+1, i+1}$ for $i=(n+1) / 2$, and suppose $s_{n+1, i}=F_{n, i}^{2} / s_{n+1, i+1}$. Thus $s_{n+1, i}$ can be calculated as:

$$
s_{n+1, i}=F_{n, i} \frac{\lambda_{n, i} F_{n, i}-\left\{e^{r \Delta t} C\left(F_{n, i}, n \triangle t\right)-\rho_{u}\right\}}{\lambda_{n, i} F_{n, i}+\left\{e^{r \Delta t} C\left(F_{n, i}, n \triangle t\right)-\rho_{u}\right\}} \quad \text { for } \quad i=(n+1) / 2
$$

where $C(K, \tau)$ is defined as in the Derman and Kani algorithm, and the $\rho_{u}$ is

$$
\rho_{u}=\sum_{j=i+1}^{n} \lambda_{n, j}\left(F_{n, j}-F_{n, i}\right)
$$

After stock prices of the initial nodes are obtained, then continue to calculate those at higher nodes $(n+1, j), j=i+2, \ldots n+1$ and transition probabilities one by one using the following recursion:

$$
s_{n+1, i+1}=\frac{s_{n+1, i}\left\{e^{r \Delta t} C\left(F_{n, i}, n \triangle t\right)-\rho_{u}\right\}-\lambda_{n, i} F_{n, i}\left(F_{n, i}-s_{n+1, i}\right)}{\left\{e^{r \Delta t} C\left(F_{n, i}, n \triangle t\right)-\rho_{u}\right\}-\lambda_{n, i}\left(F_{n, i}-s_{n+1, i}\right)},
$$

where $\rho_{u}$ is as in (8.19), $p_{n, i}$ is defined as in (8.5).

Similarly, continue to calculate the parameters iteratively at lower nodes $(n+$ 


$$
\begin{aligned}
1, j), j & =i-1, \ldots 1 . \\
s_{n+1, i} & =\frac{\lambda_{n, i} F_{n, i}\left(s_{n+1, i+1}-F_{n, i}\right)-s_{n+1, i+1}\left\{e^{r \Delta t} P\left(F_{n, i}, n \triangle t\right)-\rho_{l}\right\}}{\lambda_{n, i}\left(s_{n+1, i+1}-F_{n, i}\right)-\left\{e^{r \Delta t} P\left(F_{n, i}, n \triangle t\right)-\rho_{l}\right\}},
\end{aligned}
$$

where $\rho_{l}$ denotes the sum $\sum_{j=1}^{i-1} \lambda_{n, j}\left(F_{n, i}-F_{n, j}\right)$. Notice that in (8.13) and (8.14), $C(K, \tau)$ and $P(K, \tau)$ denote the Black-Scholes call and put option prices, this construction makes the calculation faster than the interpolation technique based on the CRR method.

The balancing inequality (8.15) and a redefinition are still used in the Barle and Cakici algorithm for avoiding arbitrage: the algorithm uses the average of $F_{n, i}$ and $F_{n, i+1}$ as the re-estimation of $s_{n+1, i+1}$.

\subsection{A Simulation and a Comparison of the SPDs}

The example used here to show the procedure of generating the IBT, is taken from Derman and Kani (1994). Assume that the current value of the stock is $S=100$, the annually compounded riskless interest rate is $r=3 \%$ per year for all time expirations, the stock has zero dividend. The annual BS implied volatility of an at-the-money call is assumed to be $\sigma=10 \%$, and the BS implied volatility increases (decreases) linearly by 0.5 percentage points with every 10 point drop (rise) in the strike. From the assumptions, we see that $\sigma_{i m p}(K, \tau)=0.15-0.0005 K$.

In order to investigate the precision of the SPD estimation obtained from the IBT, we give a simulation example assuming that the stock price process is generated by the stochastic differential equation model (8.3), with an instantaneous local volatility function $\sigma\left(S_{t}, t\right)=0.15-0.0005 S_{t}, \mu_{t}=r=0.03$. We may then easily compare the SPD estimations obtained from the two different methods.

\subsubsection{Simulation using Derman and Kani algorithm}

With the XploRe quantlet XFGIBT01.xpl, using the assumption on the BS implied volatility surface, we obtain the following one year stock price implied binomial tree, transition probability tree, and Arrow-Debreu price tree. 


\section{Q XFGIBT01.xpl}

Derman and Kani one year (four step) implied binomial tree

stock price

119.91

115.06

$110.04 \quad 110.06$

$\begin{array}{llll}105.13 & 105.13 & & \\ & 100.00 & 100.00\end{array}$

100.00

95.12

89.93

95.12

89.92

85.22

80.01

transition probability

$$
\begin{array}{cccc} 
& & & 0.60 \\
0.56 & 0.59 & 0.58 & \\
& 0.59 & 0.56 & 0.59 \\
& & 0.54 & 0.59 \\
& & & 0.59
\end{array}
$$

Arrow-Debreu price

$$
\begin{aligned}
& 0.111 \\
& 0.187 \\
& 0.327 \quad 0.312 \\
& \begin{array}{llll}
0.559 & 0.405 & \\
& 0.480 & 0.343
\end{array} \\
& \begin{array}{lllll}
1.000 & & 0.480 & & 0.343
\end{array} \\
& 0.178 \quad 0.172 \\
& 0.080 \quad 0.033
\end{aligned}
$$

This IBT is corresponding to $\tau=1$ year, and $\Delta t=0.25$ year, which shows the stock prices, and the elements at the $j$ th column are corresponding to the stock 
prices of the nodes at the $(j-1)$ th level in the tree. The second one, its $(n, j)$ element is corresponding to the transition probability from the node $(n, j)$ to the nodes $(n+1, j+1)$. The third tree contains the Arrow-Debreu prices of the nodes. Using the stock prices together with Arrow-Debreu prices of the nodes at the final level, a discrete approximation of the implied distribution can be obtained. Notice that by the definition of the Arrow-Debreu price, the risk neutral probability corresponding to each node should be calculated as the product of the Arrow-Debreu price and the factor $e^{r \tau}$.

If we choose small enough time steps, we obtain the estimation of the implied price distribution and the implied local volatility surface $\sigma_{l o c}(s, \tau)$. We still use the same assumption on the BS implied volatility surface as above here, which means $\sigma_{i m p}(K, \tau)=0.15-0.0005 K$, and assume $S_{0}=100, r=0.03, T=5$ year.

Q XFGIBT02.xp1

Two figures are generated by running the quantlet XFGIBT02 .xpl, Figure 8.2 shows the plot of the SPD estimation resulting from fitting an implied five-year tree with 20 levels. The implied local volatilities $\sigma_{l o c}(s, \tau)$ in the implied tree at different time to maturity and stock price levels is shown in Figure 8.3, which obviously decreases with the stock price and increases with time to maturity as expected.

\subsubsection{Simulation using Barle and Cakici algorithm}

The Barle and Cakici algorithm can be applied in analogy to Derman and Kani's. The XploRe quantlets used here are similar to those presented in Section 8.2.1, one has to replace the quantlet IBTdk by IBTdc. The following figure displays the one-year (four step) stock price tree, transition probability tree, and Arrow-Debreu tree. Figure 8.4 presents the plot of the estimated SPD by fitting a five year implied binomial tree with 20 levels to the volatility smile using Barle and Cakici algorithm, and Figure 8.5, shows the characteristics of the implied local volatility surface of the generated IBT, decreases with the stock price, and increases with time. 


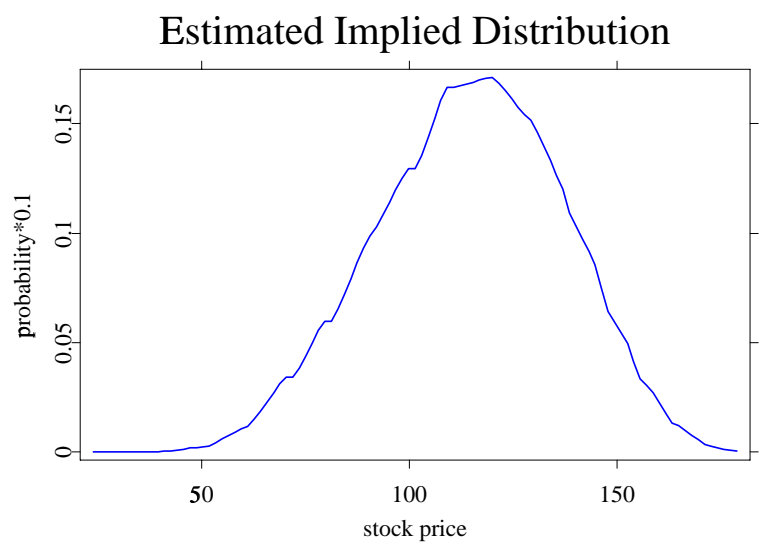

Figure 8.2. SPD estimation by the Derman and Kani IBT.

Implied Local Volatility Surface

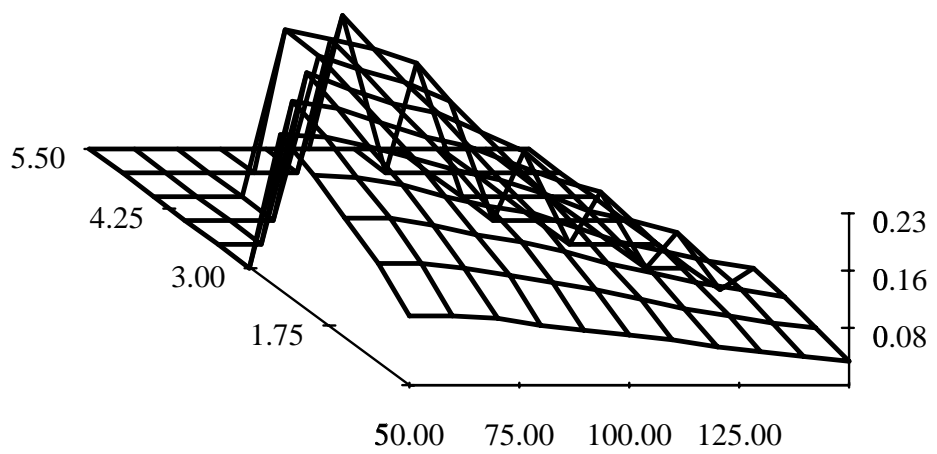

Figure 8.3. Implied local volatility surface estimation by the Derman and Kani IBT.

Barle and Cakici one year implied binomial tree

stock price 


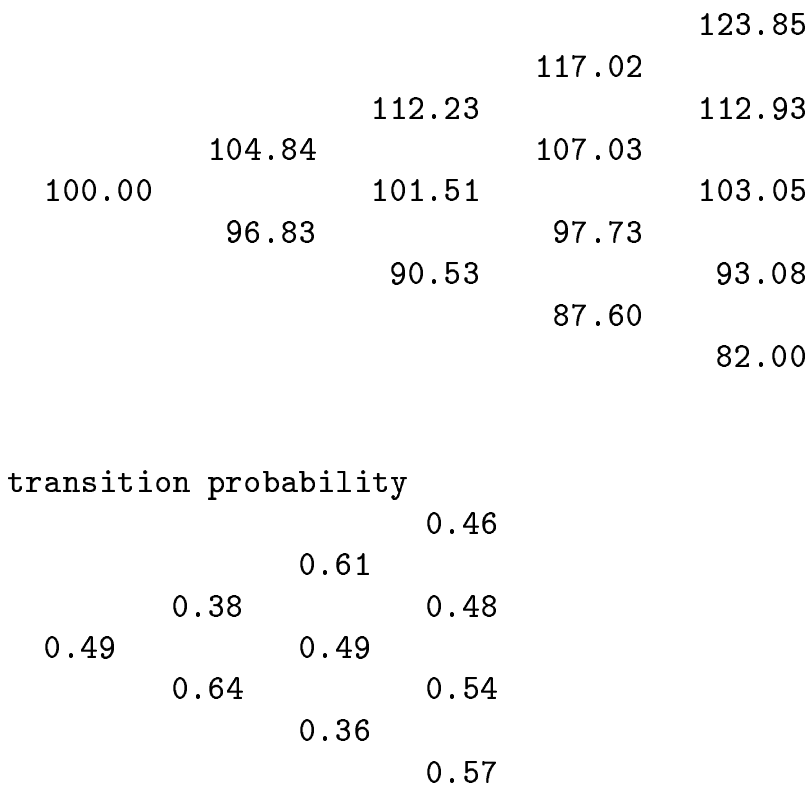

Arrow-Debreu price

$$
\begin{aligned}
& 0.050 \\
& \begin{array}{lll}
0.185 & 0.111 & \\
& & 0.240
\end{array} \\
& 0.486 \\
& \begin{array}{ll}
0.373 & 0.394
\end{array} \\
& 1.000 \\
& \begin{array}{ll}
0.619 & 0.378
\end{array} \\
& \begin{array}{lll}
0.181 & 0.237
\end{array} \\
& \begin{array}{ll}
0.116 & 0.050
\end{array}
\end{aligned}
$$

\subsubsection{Comparison with Monte-Carlo Simulation}

We now compare the SPD estimation at the fifth year obtained by the two IBT methods with the estimated density function of the Monte-Carlo simulation of $S_{t}, t=5$ generated from the model $(8.3)$, where $\sigma\left(S_{t}, t\right)=0.15-0.0005 S_{t}$, 


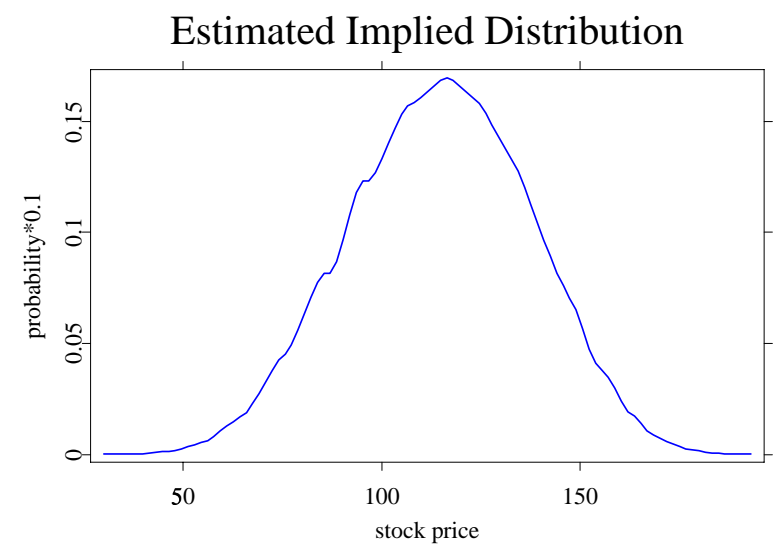

Figure 8.4. SPD estimation by the Barle and Cakici IBT.

\section{Implied Local Volatility Surface}

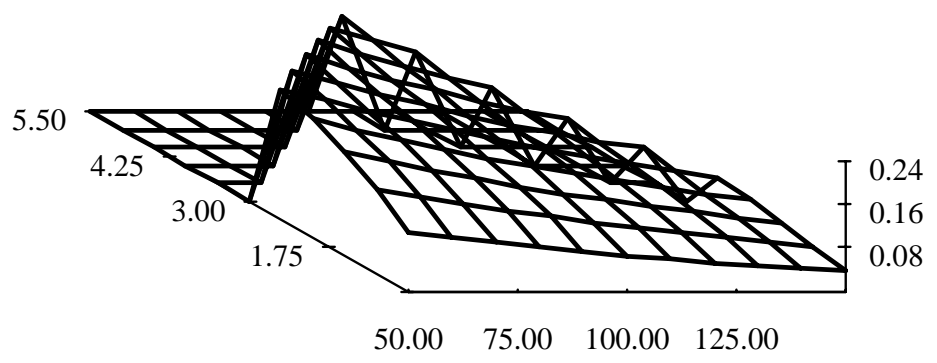

Figure 8.5. Implied local volatility surface by the Barle and Cakici IBT.

$\mu_{t}=r=0.03$. We use the Milstein scheme, Kloeden, Platen and Schurz (1994) to perform the discrete time approximation in (8.3). It has strong convergence rate $\delta^{1}$. We have set the time step with $\delta=1 / 1000$ here. 
In order to construct the IBT, we calculate the option prices corresponding to each node at the implied tree according to their definition by Monte-Carlo simulation.

Q XFGIBT03.xpl QXXFIBTcdk.xpl Q XFGIBTcbc.xpl

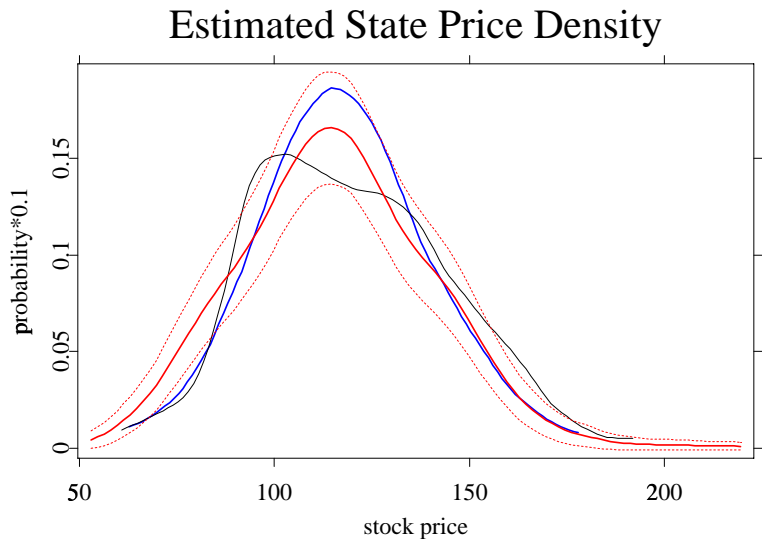

Figure 8.6. SPD estimation by Monte-Carlo simulation, and its $95 \%$ confidence band, the B \& C IBT, from the D \& K IBT (thin), level $=20, T=5$ year, $\Delta t=0.25$ year.

Here we use the quantlets IBTcdk and IBTcbc (which are included in XFGIBTcdk.xpl and XFGIBTcbc.xpl respectively), these two are used to construct the IBT directly from the option price function, not starting from the BS implied volatility surface as in quantlets IBTdk and IBTbc. In the data file "IBTmcsimulation20. dat", there are 1000 Monte-Carlo simulation samples for each $S_{t}$ in the diffusion model (8.3), for $t=i / 4$ year, $i=1, \ldots 20$, from which we calculate the simulated values of the option prices according to its theoretical definition and estimate the density of $S_{t}, T=5$ year as the SPD estimation at the fifth year.

From the estimated distribution shown in the Figures 8.2.3, we observe their deviation from the log-normal characteristics according to their skewness and kurtosis. The SPD estimation obtained from the two IBT methods coincides with the estimation obtained from the Monte-Carlo simulation well, the difference between the estimations obtained from the two IBTs is not very large. 
On the other hand, we can also estimate the implied local volatility surface from the implied binomial tree, and compare it with the one obtained by the simulation. Compare Figure 8.7 and Figure 8.8 with Figure 8.9, and notice that in the first two figures, some edge values cannot be obtained directly from the five-year IBT. However, the three implied local volatility surface plots all actually coincide with the volatility smile characteristic, the implied local volatility of the out-the-money options decreases with the increasing stock price, and increase with time.

\section{Implied Local Volatility Surface}

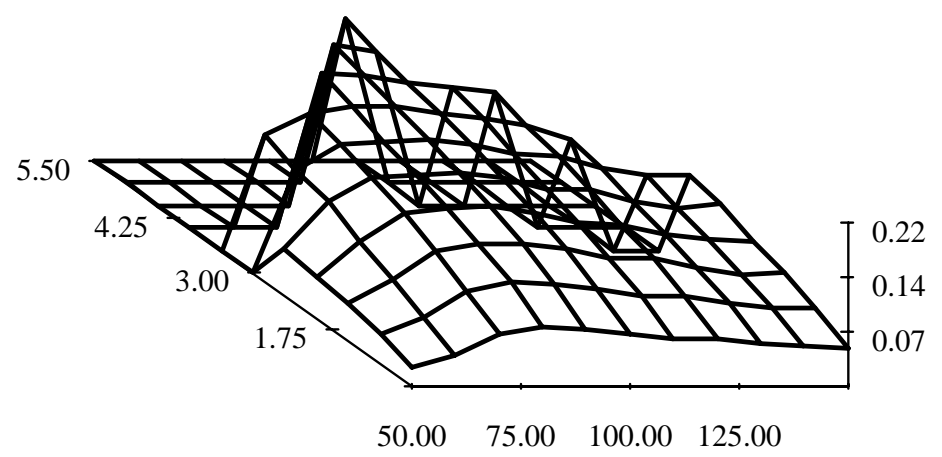

Figure 8.7. Implied local volatility surface of the simulated model, calculated from D\& K IBT.

We use the data file "IBTmcsimulation50.dat" to obtain an estimated BS implied volatility surface. There are 1000 Monte-Carlo simulation samples for each $S_{t}$ in the diffusion model (8.3), for $t=i / 10$ year in it, $i=1, \ldots 50$, because we can calculate the BS implied volatility corresponding to different strike prices and time to maturities after we have the estimated option prices corresponding to these strike price and time to maturity levels. Figure 8.10 shows that the BS implied volatility surface of our example reflects the characteristics that the BS implied volatility decrease with the strike price. But this BS implied volatility surface does not change with time a lot, which is probably due to our assumption about the local instantaneous volatility function, which only changes with the stock price. 


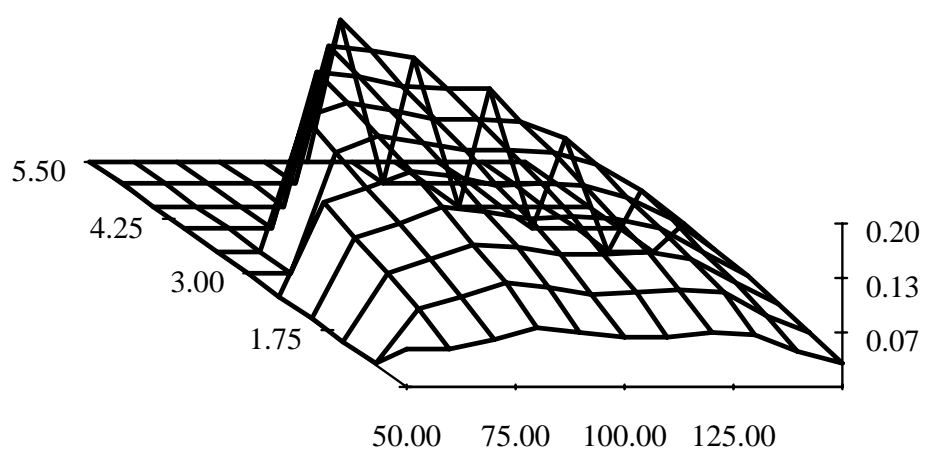

Figure 8.8. Implied local volatility surface of the simulated model, calculated from B\& C IBT.

Q XFGIBT04.xpl

\subsection{Example - Analysis of DAX data}

We now use the IBT to forecast the future price distribution of the real stock market data. We use DAX index option prices data at January 4, 1999, which are included in $\mathrm{MD}^{*} \mathrm{BASE}$, a database located at CASE (Center for Applied Statistics and Economics) at Humboldt-Universität zu Berlin, and provide some dataset for demonstration purposes. In the following program, we estimate the BS implied volatility surface first, while the quantlet volsurf, Fengler, Härdle and Villa (2001), is used to obtain this estimation from the market option prices, then construct the IBT using Derman and Kani method and calculate the interpolated option prices using CRR binomial tree method. Fitting the function of option prices directly from the market option prices is hardly ever attempted since the function approaches a value of zero for very high strike 


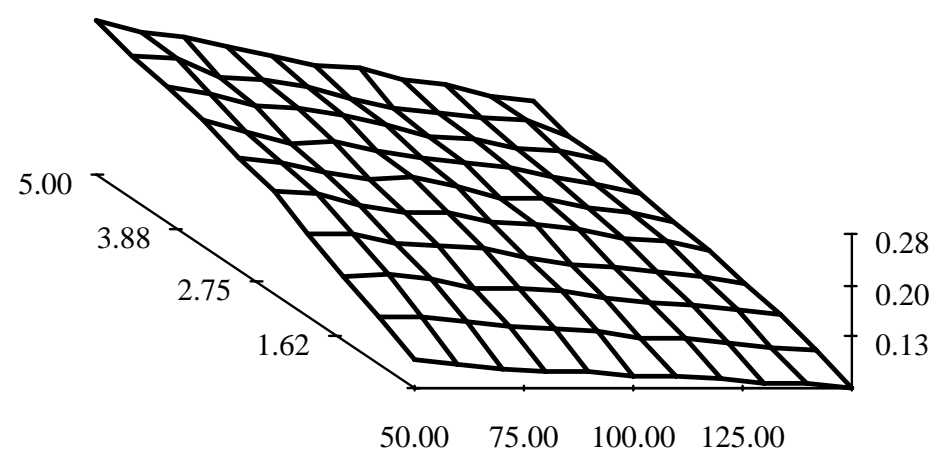

Figure 8.9. Implied local volatility surface of the simulated model, calculated from Monte-Carlo simulation.

prices and option prices are bounded by non-arbitrage conditions.

Q. XFGIBT05.xpl

Figure 8.11 shows the price distribution estimation obtained by the Barle and Cakici IBT, for $\tau=0.5$ year. Obviously, the estimated SPD by the Derman and Kani IBT can be obtained similarly. In order to check the precision of the estimated price distribution obtained by the IBT method, we compare it to use DAX daily prices between January 1, 1997, and January 4. 1999. The historical time series density estimation method described in Aï-Sahalia, Wang and Yared (2000) is used here. Notice that Risk-neutrality implies two kinds of SPD should be equal, historical time series SPD is in fact the conditional density function of the diffusion process. We obtain the historical time series SPD estimation by the following procedure:

1. Collect stock prices time series 


\section{Volatility Surface}

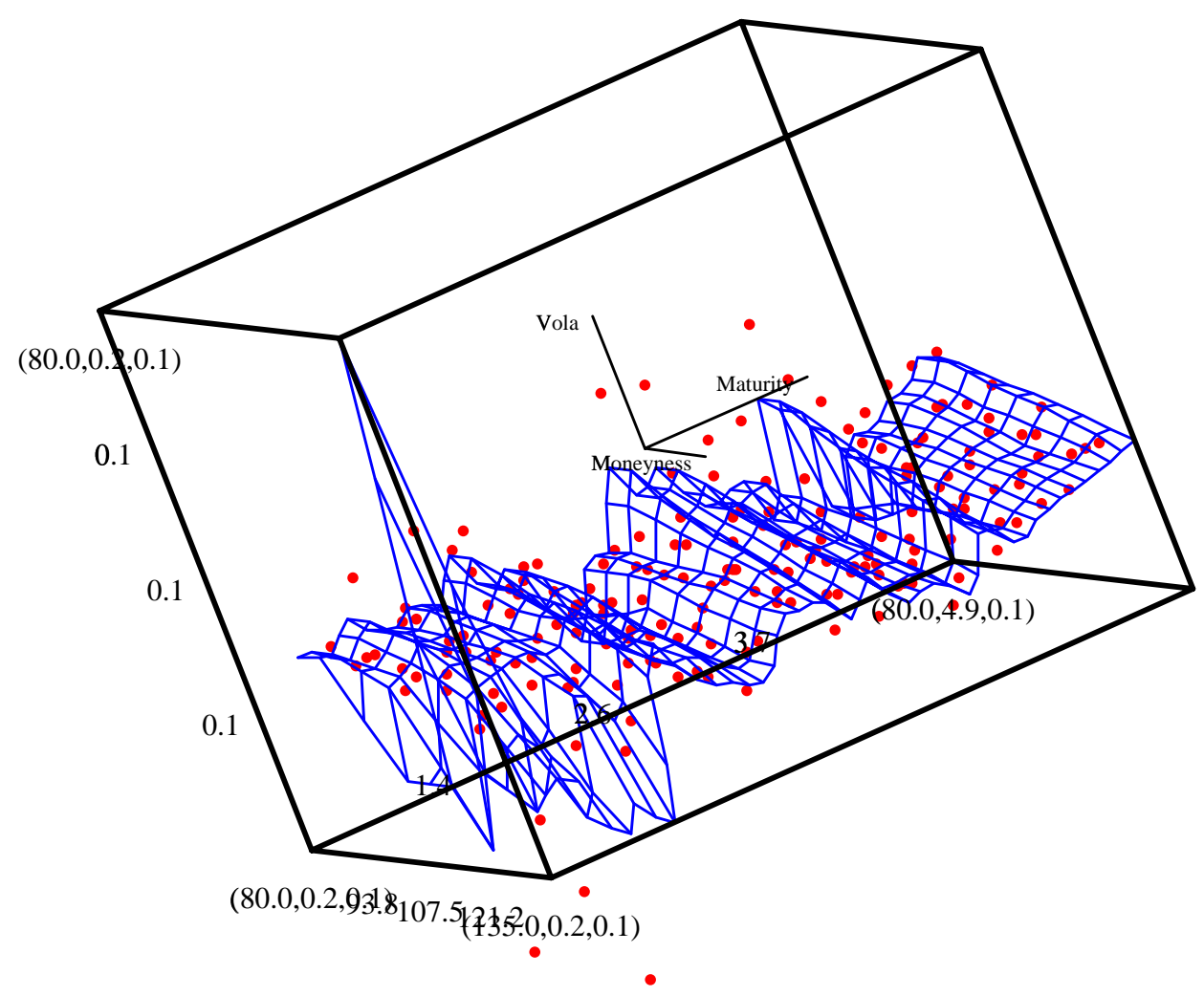

Figure 8.10. BS implied volatility surface estimation by Monte-Carlo simulation.

2. Assume this time series is a sample path of the diffusion process

$$
\frac{d S_{t}}{S_{t}}=\mu_{t} d t+\sigma\left(S_{t}, t\right) d Z_{t},
$$




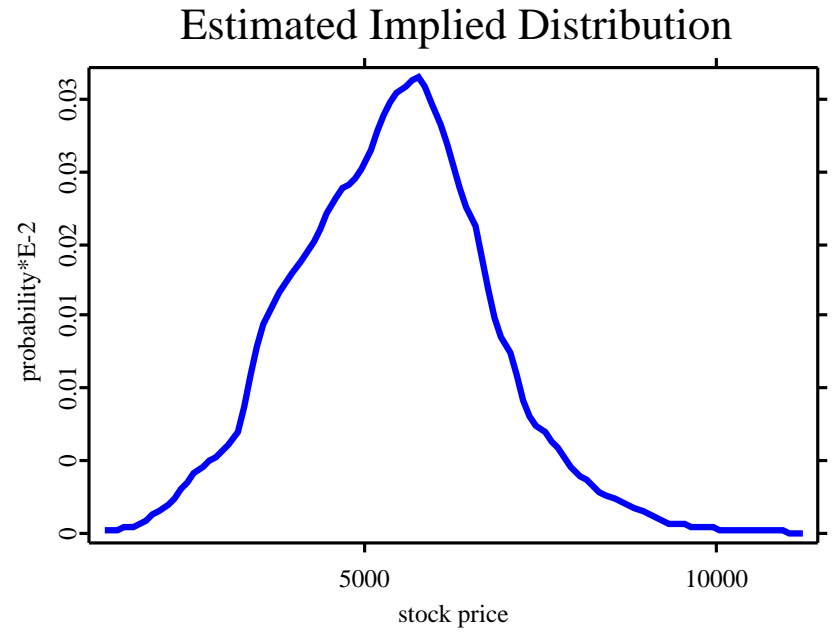

Figure 8.11. The estimated stock price distribution, $\tau=0.5$ year.

where $d Z_{t}$ is a Wiener process with mean zero and variance equal to $d t$.

3. Estimate diffusion function $\sigma(\cdot, \cdot)$ in the diffusion process model using nonparametric method from stock prices time series

4. Make Monte-Carlo simulation for the diffusion process with drift function is interest rate and estimated diffusion function

5. Estimate conditional density function $g=p\left(S_{T} \mid S_{t}, \hat{\mu}, \hat{\sigma}\right)$ from MonteCarlo simulated process

From Figure 8.12 we conclude that the SPD estimated by the Derman and Kani IBT and the one obtained by Barle and Cakici IBT can be used to forecast future SPD. The SPD estimated by different methods sometimes have deviations on skewness and kurtosis. In fact the detection of the difference between the historical time series SPD estimation and the SPD recovered from daily option prices may be used as trading rules, see Table 8.1 and Chapter 10. In Table 8.1, SPD estimated from daily option prices data set is expressed by $f$ and the time series SPD is $g$. A far out of the money (OTM) call/put is defined as one whose exercise price is $10 \%$ higher (lower) than the future price. While a near OTM call/put is defined as one whose exercise price is $5 \%$ higher (lower) 


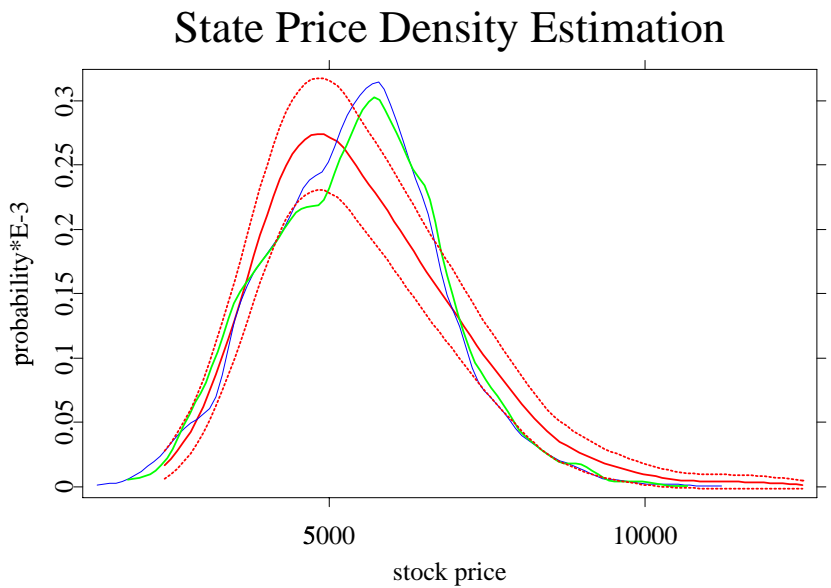

Figure 8.12. SPD estimation by three methods, by historical estimation, and its $95 \%$ confidence band (dashed), by B \& C IBT, and by D $\& \mathrm{~K}$ IBT (thin), $\tau=0.5$ year.

but $10 \%$ lower(higher)than the future price. When skew $(f)<\operatorname{skew}(g)$, agents apparently assign a lower probability to high outcomes of the underlying than would be justified by the time series SPD (see Figure 8.13). Since for call options only the right 'tail' of the support determines the theoretical price the latter is smaller than the price implied by diffusion process using the time series SPD. That is we buy calls. The same reason applies to put options.

\begin{tabular}{|c|c|c|c|}
\hline \multicolumn{4}{|c|}{ Trading Rules to exploit SPD differences } \\
\hline Skewness & $(S 1)$ & skew $(f)<\operatorname{skew}(g)$ & sell OTM put, \\
\hline Trade & & $\operatorname{skew}(f)>\operatorname{skew}(g)$ & $\begin{array}{l}\text { buy OTM call } \\
\text { buy OTM put }\end{array}$ \\
\hline Kurtosis & $(K 1)$ & $\operatorname{kurt}(f)>\operatorname{kurt}(g)$ & sell far OTM and ATM \\
\hline Trade & $(K 2)$ & $\operatorname{kurt}(f)<\operatorname{kurt}(g)$ & $\begin{array}{l}\text { buy near OTM options } \\
\text { buy far OTM and ATM, } \\
\text { sell near OTM options }\end{array}$ \\
\hline
\end{tabular}

From the simulations and real data example, we find that the implied binomial tree is an easy way to assess the future stock prices, capture the term structure 


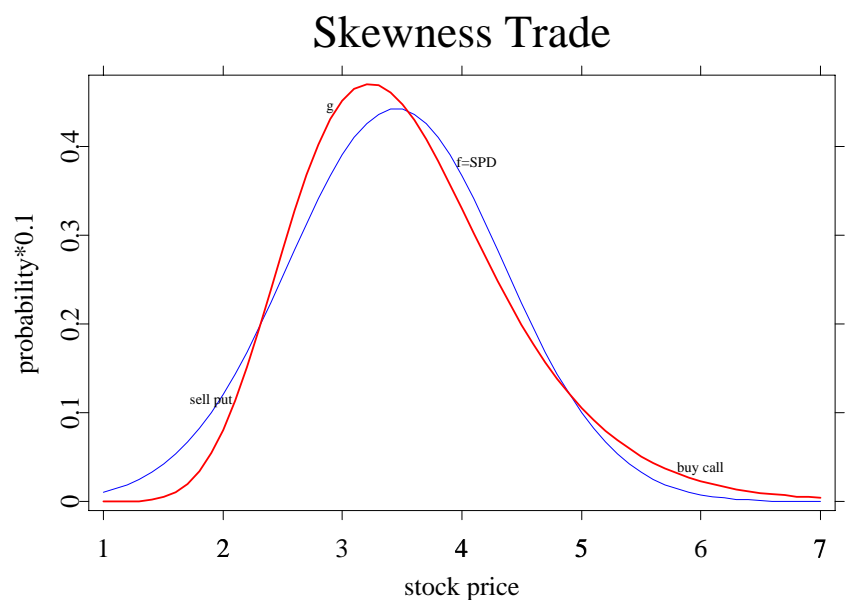

Figure 8.13. Skewness Trade, skew $(f)<\operatorname{skew}(g)$.

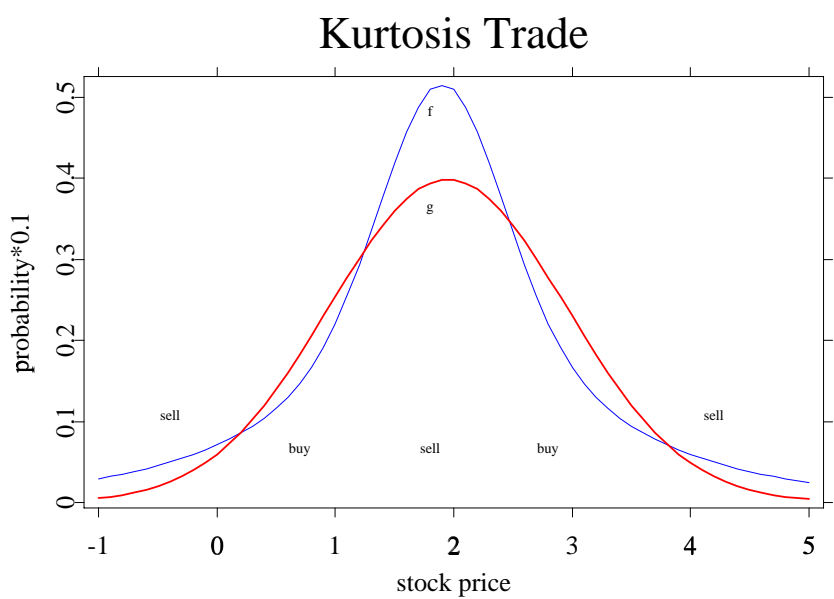

Figure 8.14. Kurtosis Trade, $\operatorname{kurt}(f)>\operatorname{kurt}(g)$.

of the underlying asset, and replicate the volatility smile. But the algorithms still have some deficiencies. When the time step is chosen too small, negative transition probabilities are encountered more and more often. The modification of these values loses the information about the smile at the corresponding 
nodes. The Barle and Cakici algorithm is a better choice when the interest rate is high.Figure 8.15 shows the deviation of the two methods under the situation that $r=0.2$. When the interest rate is a little higher, Barle and Cakici algorithm still can be used to construct the IBT while Derman and Kani's cannot work any more. The times of the negative probabilities appear are fewer than Derman and Kani construction (see Jackwerth (1999)).

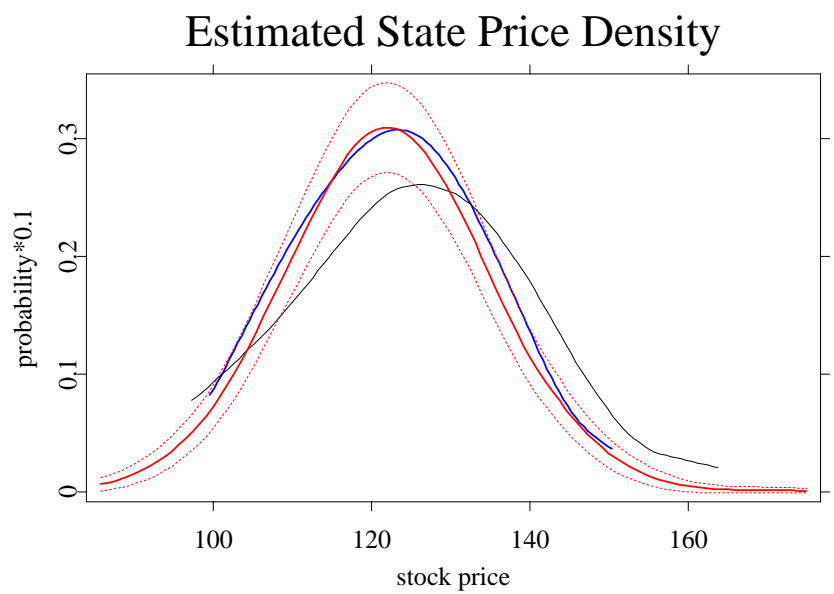

Figure 8.15. SPD estimation by Monte-Carlo simulation, and its $95 \%$ confidence band (dashed), the B \& C IBT, from the D \& K IBT (thin), level $=20, \tau=1$ year, $r=0.20$.

Besides its basic purpose of pricing derivatives in consistency with the market prices, IBT is useful for other kinds of analysis, such as hedging and calculating of implied probability distributions and volatility surfaces. It estimate the future price distribution according to the historical data. On the practical application aspect, the reliability of the approach depends critically on the quality of the estimation of the dynamics of the underlying price process, such as BS implied volatility surface obtained from the market option prices.

The IBT can be used to produce recombining and arbitrage-free binomial trees to describe stochastic processes with variable volatility. However, some serious limitations such as negative probabilities, even though most of them appeared at the edge of the trees. Overriding them causes loss of the information about the smile at the corresponding nodes. These defects are a consequence of the requirement that a continuous diffusion is approximated by a binomial process. Relaxation of this requirement, using multinomial trees or varinomial trees is 
possible.

\section{Bibliography}

Aït-Sahalia, Y. and Lo, A. (1998). Nonparametric Estimation of State-Price Densities Implicit in Financial Asset Prices, Journal of Finance, 53: 499547.

Aït-Sahalia, Y. , Wang, Y. and Yared, F.(2001). Do Option Markets Correctly Price the Probabilities of Movement of the Underlying Asset? Journal of Econometrics, 102: 67-110.

Barle, S. and Cakici, N. (1998). How to Grow a Smiling Tree The Journal of Financial Engineering, 7: 127-146.

Bingham, N.H. and Kiesel, R. (1998). Risk-neutral Valuation: Pricing and Hedging of Financial Derivatives, Springer Verlag, London.

Cox, J., Ross, S. and Rubinstein, M. (1979). Option Pricing: A simplified Approach, Jouranl of Financial Economics 7: 229-263.

Derman, E. and Kani, I. (1994). The Volatility Smile and Its Implied Tree http://www.gs.com/qs/

Derman, E. and Kani, I. (1998). Stochastic Implied Trees: Arbitrage Pricing with Stochastic Term and Strike Structure of Volatility, International Journal of Theroetical and Applied Finance 1: 7-22.

Dupire, B. (1994). Pricing with a Smile, Risk 7: 18-20.

Fengler, M. R., Härdle, W. and Villa, Chr. (2001). The Dynamics of Implied Volatilities: A Common Principal Components Approach, Discussion paper 38, Sonderforschungsbereich 373, Humboldt-Universität zu Berlin.

Härdle, W., Hlávka, Z. and Klinke, S. (2000). XploRe Application Guide, Springer Verlag, Heidelberg.

Härdle,W. and Yatchew, A. (2001). Dynamic Nonparametric State price Density Estimation using Constrained least Squares and the Bootstrap, Discussion paper 1, Sonderforschungsbereich 373 , Humboldt-Universität zu Berlin. 
Hull, J. and White, A. (1987). The Pricing of Options on Assets with Stochastic Volatility, Journal of Finance 42: 281-300.

Jackwerth, J. (1999). Optional-Implied Risk-Neutral Distributions and Implied Binomial Trees: A Literature Review, Journal of Finance 51: 1611-1631.

Jackwerth, J. and Rubinstein, M. (1996). Recovering Probability Distributions from Option Prices, Journal of Finance 51: 1611-1631.

Kloeden, P., Platen, E. and Schurz, H. (1994). Numerical Solution of SDE Through Computer Experiments, Springer Verlag, Heidelberg.

Merton, R. (1976). Option Pricing When Underlying Stock Returns are Discontinuous, Journal of Financial Economics January-March: 125-144.

Rubinstein, M. (1994). Implied Binomial Trees. Journal of Finance 49: 771818. 